Saudi Journal of Oral and Dental Research

Abbreviated Key Title: Saudi J Oral Dent Res

ISSN 2518-1300 (Print) |ISSN 2518-1297 (Online)

Scholars Middle East Publishers, Dubai, United Arab Emirates

Journal homepage: http://scholarsmepub.com/sjodr/

Review Article

\title{
Insights into Epigenetics Mechanisms in Oral Squamous Cell Carcinoma
}

\author{
Loulwah Alothman ${ }^{1}$, May Alsenani ${ }^{1}$, Reem Alrabiah ${ }^{1}$, Amjad Abu Ras ${ }^{2}$, Ethar Abulhassan ${ }^{2 *}$, Rana Aldayel ${ }^{2}$, Rania \\ Almutairi ${ }^{2}$, Rawan Alsaif ${ }^{2}$ \\ ${ }^{1}$ Faculty in oral medicine department, College of dentistry, King Saud University Saudi Arabia \\ ${ }^{2}$ Dental Intern, King Saud University, Riyadh, Saudi Arabia
}

DOI: $10.36348 /$ sjodr.2020.v05i01.005

| Received: 02.01.2020 | Accepted: 12.01.2020 | Published: 18.01 .2020

*Corresponding author: Ethar Abulhassan

\section{Abstract}

Oral squamous cell carcinoma (OSCC) is one of the most common oral cancers accounting for $96 \%$ of all head and neck cancers. Beside the different endogenous and environmental risk factors, epigenetics play an important role in causing OSCC. Epigenetics shed light on the presence of inherited changes in the phenotype of a gene with the absence of an alteration in the DNA sequence. The epigenetic modifications can result in the emersion of cancer, autoimmune diseases, aging and other diseases. Under several circumstances, DNA undergoes methylation. This methylation might be related to normal development or serious diseases like cancer. DNA methylation is a heritable biochemical modification occurs predominantly by transferal of a methyl group to $\mathrm{C}$ - 5 position of the cytosine ring (CpGs) which may cause epigenetic disturbances. This review aims toward investigating the genetics in literature to help in diagnosing OSCC with new modalities that are less invasive compared to histopathology. Studied genes including ECAD, DAPK, MGMT and P53 has different methylation frequencies in relation to OSCC which may be related to their significant role in early diagnosis and prognosis. Since genetics has a major role in OSCC, we searched most recently reported genes and technologies seeking early detection and treatment of OSCC results in a better prognosis and more conservative treatment.

Keywords: Epigenetics, Oral Cancer, (OSCC), endogenous.

Copyright @ 2020: This is an open-access article distributed under the terms of the Creative Commons Attribution license which permits unrestricted use, distribution, and reproduction in any medium for non-commercial use (NonCommercial, or CC-BY-NC) provided the original author and source are credited.

\section{INTRODUCTION}

Oral squamous cell carcinoma (OSCC) is one of the most common cancers of the oral cavity[1]. It accounts for $96 \%$ of all head and neck cancers[2]. 100,000 patients die annually from OSCC and the incidence is increasing to 500,000 worldwide[2]. With all the innovations in diagnosing and treating OSCC, a percentage of $50 \%$ and more of patients suffer from relapse and increased mortality rate[2]. Different endogenous and environmental risk factors related to OSCC were discussed in the literature. Risk factors could involve tobacco, alcohol consumption, viral infections (HPV) and chronic inflammation[1, 3, 4]. Moreover, epigenetics play an important role in causing oral squamous cell carcinoma (OSCC). Epigenetics can be defined as the study of all heritable changes in gene phenotype and expression which are not coded in the DNA sequence[2, 5, 6]. Epigenetic alterations can cause major changes in the tumor progression, metastasis and response to chemo-radiotherapy[6]. The previously mentioned predisposing factors might act synergistically to a wide range of genetic and epigenetic events leading to suppression of tumor suppressor genes by promoting hyper-methylation and activation of OSCC oncogenesis[4]. The objective of this review is to review and investigate the genetics in literature to help in diagnosing OSCC with new modalities that are less invasive compared to histopathology.

\section{Epigenetics}

Epigenetics explain the inherited changes in the phenotype of a gene with the absence of an alteration in the DNA sequence[7]. Since the discovery of tumor suppressor genes silencing with the lack of any genetic modifications, the interest toward epigenetics has been raised dramatically[7]. Epigenetics has brought a new conception in the development of organisms, their genetics and evolution[7]. The expression of genes can be affected by environmental factors such as stress, nutritional factors, infections and toxins[7]. These factors can start altering the gene expression of a creature from his intrauterine life and continues throughout the rest of his life[7]. The epigenetic modifications can affect a wide variety of genes including tumor suppressor genes that become 
silenced in malignant tumor cells[7]. Changes in epigenetics can accumulate in the cells resulting in the emersion of cancer, autoimmune diseases, aging and other diseases[7].
"Hall marks of cancer" are six main characteristics that are found in most and perhaps all cancer tumors which are (Fig. 1)[8]:

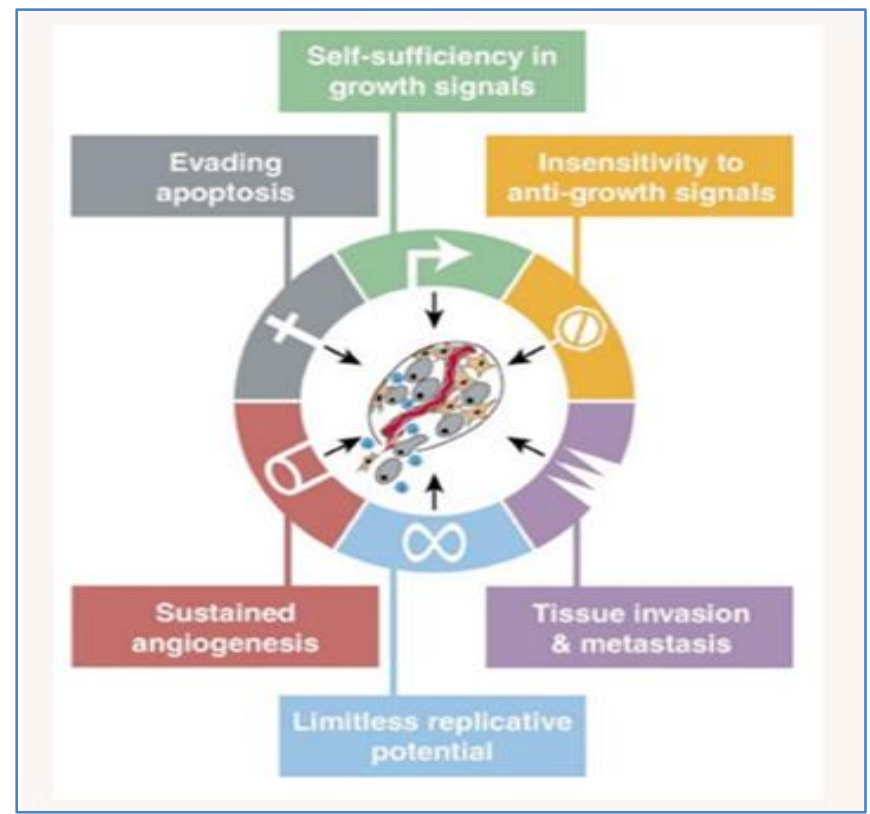

Fig-1: [8] "Hall marks of cancer".

\section{Malformations in anti-growth signals}

Growth inhibitory signals work on normal cells to preserve balance in the body tissues[7]. These signals can interfere with the growth by two methods; either by knocking out the cells from the cycle of proliferation to an inactive state which might be induced later and reactivated[7]. Otherwise, cells tempt to abandon their potential of proliferation[7].

\section{Acquisition of stimulating signals for self-sufficient growth}

Normal cells require the presence of mitotic growth signals for their replication and proliferation[7]. In contrast, tumor cells produce their own growth signals reducing their need to the exogenous stimulatory factors[7]. The lack of growth factors dependence in the tumor cells leads to disturbance in the body homeostasis[7].

\section{Avoidance of apoptosis}

The growth of tumor is guided by the uncontrolled cell proliferation and loss of the ability of cell cessation[7, 9]. Apoptosis -programmed cell deathis a normal physiological process that protects the tissues from abnormal cell growth[7, 9]. Apoptosis is kept in a silent format until abnormality is detected inducing the program cell death to destroy the abnormal cells[7, 9]. It takes 30-120 minutes for cell degradation and 24 hours for engulfing the cell and clearing the tissue area[7,9].

\section{Sustained Angiogenesis}

Cells depend on vasculature for their nutrition and oxygenation which is critical for their function and persistence [7, 10-12]. Angiogenesis is controlled precisely and proliferative cells have the capability to promote growth of blood vessels [7, 10-12]. Tumors with unusual growth pattern -at first- lack the ability of angiogenesis which is considered the key for their growth and increase in size [7, 10-12].

\section{The ability for invasion and metastasis}

During the process of most cancer tumors, cells of primary tumors start invading adjacent tissues, and later traveling to distant organs where they can repopulate to form a new cancer tumor[7, 13].

\section{Replicative ability without limitation}

It has been mentioned before that tumor cells have the ability to escape apoptosis, to gain self-growth signals and to resist growth inhibitory signals, which give them the ability to proliferate infinitely[7, 14].

The principal epigenetic processes included in cancer formation are DNA methylation, histone modification and small non-coding RNAs (ncRNA)[6]. This review will discuss mainly DNA methylation as it is the most studied one.

\section{DNA Methylation}

Despite the huge variation in structure and function, all cells in our body contain the same DNA sequence. DNA under several circumstances and several environmental stresses undergo DNA 
methylation before and after birth[7]. This methylation is essential for normal development, where it plays an important role in number of processes including embryonic stem cells differentiation. However, when altered or disrupted it contributes to serious diseases like cancer[3]. As mentioned previously DNA methylation is one of the mechanisms that are responsible for epigenetic regulation, gene silencing as well as chromatin architecture and function modification[4, 7]. Among all mechanisms, DNA methylation is the most intensely studied epigenetic change in mammalian DNA[7]. These epigenetic changes may persist throughout life or in some cases it is reversible[7]. DNA methylation is a heritable biochemical modification occurs predominantly by transferal of a methyl group to $\mathrm{C}-5$ position of the cytosine ring (CpGs) (Fig. 2.a)[15, 3,7]. The methyl group attached to Cytosine dinucleotide will protrude into the DNA groove displacing the transcription factor from the DNA, with the help of histone modification and deacetylation a chromatin condensation will happen, thus preventing the access of gene expression factor resulting in gene silencing (Fig. 2.b)[7]. CpGs dinucleotide areas are underrepresented region in the genome that most frequently appears in the promoter region and in the first exon of specific gene[7]. More than $98 \%$ of DNA methylation occurs in CpG dinucleotides of somatic cells while around $25 \%$ of methylation appears in a non-CpG in embryonic stem cells[3]. DNA methylation is carried out by a group of enzymes called methyltransferases (DNMTs). These enzymes break down into; DNMT1, DNMT2, DNMT3a, DNMT3b and DNMT3L[16]. These enzymes start the mechanism of methylation with the help of S-adenosyl methionine (SAM) as a methyl group donor[7].

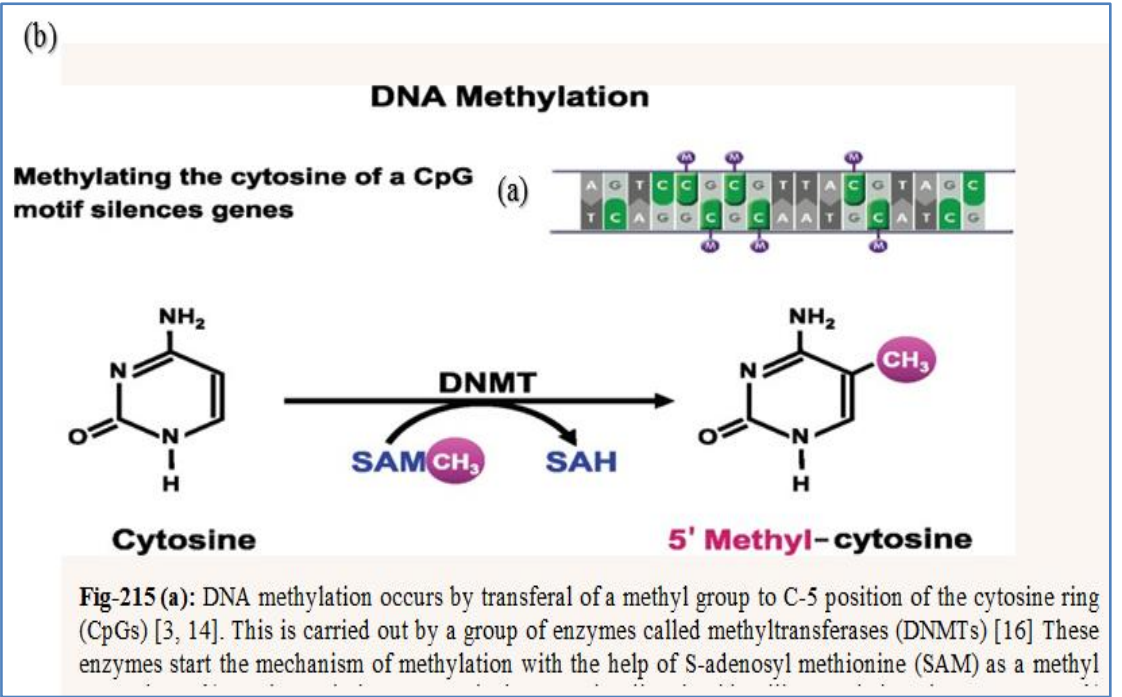

When comparing a sample from normal tissue with another from a tumor of oral cancer, increased rates of hypoldemethylation and hypermethylation of promoter regions are apparent in the tumor samples[4]. DNA methylation involves two different mechanisms; hyper and hypoldemethylation. Hypoldemethylation causes chromosome instability by releasing repetitive elements within the genome, activation of silenced proto-oncogenes that are already hypermethylated, increases the mutation rates as well as being considered an early hallmark of tumor cells, this can be seen in smokers' oral tissue[3, 4]. Hypermethylation acts on CpG site by adding methyl group thus preventing the access of gene expression factors resulting in gene silencing as well as contributing in cancer progression, this can be seen in alcohol consumers' oral tissues[3]. It has been shown that approximately $5-10 \%$ of the $\mathrm{CpG}$ islands are methylated in malignant pathology[4].

Multiple analysis of specific gene hypermethylation revealed that epigenetic status can be used as prognostic tool or parameter for overall survival in cases of OSCC[4]. Each gene is hypermethylated in different pattern and frequency which helps in understanding the molecular initiation and OSCC progression[2]. Some of the reported genes in the literature are:

\section{ECAD (CDH; E-cadherin)}

It is located on chromosome 16[3]. Riethmacher et al.1995 stated that it is a glycoprotein that plays an essential role in the formation and development of epithelial cells during embryonic stages and later in life[14]. Furthermore, It participates in forming an adhesive junction in the epithelium[3]. This molecule plays an important role in the formation and preservation of cellular and intercellular adhesion, cell polarity, intracellular signaling and tissue architecture $[3,4,7]$. ECAD was additionally reported in regulating tumor invasion[5, 7]. In a study done by $\mathrm{S}$. Dvojakovsa et al. reported a frequency of hypermethylation in ECAD gene ranging from 27\%$91 \%$. His study discussed different genes in OSCC and he found that ECAD was the most commonly 
methylated gene between them in tumor samples with a percentage of $90 \%$ [2]. Moreover, he reported that ECAD is the most significant predictive marker of oral cancer initiation. An important mentioned supporting factor is the absence of ECAD hypermethylation in normal samples and the lesser hypermethylation in normal contralateral tissues $(89,7 \%)$ in OSCC patients[2]. This highly supports the diagnostic effectiveness of this gene as a promising tool and biomarker in the future for diagnosing early OSCC having the highest accuracy in the study[2]. Thus, identification of DNA methylation could be used as a screening method for OSCC[2]. Any reduction in ECAD expression would result in tumor invasion and potentially metastasis of carcinomas[5]. Methylation of ECAD could be apparent in precancerous stages [5].

K.N. Hema et al. reported in his study that tissue metastasis is related to an alteration in ECAD gene expression[3]. Moreover, low expression levels of ECAD is related to more aggressive behavior of OSCC and poor clinical prognosis [3, 4]. It is hypermethylated in $7 \%-46 \%[3,4,7]$. ECAD methylation was linked to poor survival, too[7].

\section{DAPK (death-associated protein kinase)}

It is located on chromosome 9, encoding for apoptosis[3, 4]. DAPK functions normally as a programmed cell death mediator which occurs under the effect of gamma interferon [9]. Its hypermethylation frequency was reported as $18-27 \%$ and reaching up to $68.3 \%$ in other studies[3, 7]. The previously mentioned study by $\mathrm{S}$. Dvojakovsa have discussed DAPK as well[2]. He reported a frequency by which DAPK was hypermethylated in $18 \%-75.3 \%$ [2]. DAPK was mentioned as one of the most frequently detected genes in OSCC by a percentage of 75\%[2]. DAPK has a lower results in normal tissues which might be contributed to its role in advanced or late pathogenesis of OSCC[2]. It is important to note that DAPK is not useful in early detection and diagnosis of OSCC[2]. However, this does not exclude its importance in the diagnosis of OSCC since it showed a reasonably very high diagnostic accuracy[2]. A study done by A.I.Irimie reported that DAPK can be used as a marker for overall survival individually[4].

\section{MGMT (06-methylgluanine-DNA methyl transferase):}

This gene is located on chromosome 10[3]. MGMT is an enzyme that helps in DNA repairing process[3]. It encodes a protein which functions to help in DNA repair involved in cellular defense against mutagenesis and toxicity[11]. The frequency of MGMT gene hypermethylation published in the literature is $7 \%$ $68 \%[2,3]$. Dvojakovska et al. reported in his study that MGMT was one of the most often methylated genes in tumor sample with a percentage of $75 \%$ [2]. He also reported a diagnostic accuracy of $83.3 \%$ for MGMT gene in a true positive OSCC[2]. However, in normal contralateral tissues, the diagnostic value was about 83.1\%[2]. Comparing MGMT to ECAD and DAPK, these genes were hypermethylated in all tumor sample and normal contralateral samples ranked as following: ECAD,MGMT then DAPK[2]. MGMT together with ECAD were considered as the most significant markers to predict OSCC carcinogenesis[2].

\section{4- p53}

This gene is located on chromosome 17[3]. It encode a tumor suppressor protein[3]. p53 gene is responsible for many principle processes as a reaction to cellular stresses including cell-cycle progression, cellular differentiation, DNA repair and apoptosis while it remains inactivated in unstressed cells $[3,12]$. As mentioned before, the gene is an example for the first hall marks of cancer which is abnormalities in growth inhibitory signals[7]. p53 start to function when stress occurs, wither it was endogenous or exogenous. Its mechanism is blocking the cell cycle by elevating its levels in response to stress and eventually allowing DNA- repair[3]. When p53 function in different manner, the ability of the cell to respond to stress will be different, leading to genomic instability[3]. Studies reported a mutation in $\mathrm{p} 53$ gene in a wide range of cancers through the hole body[3, 7]. Regarding oral cancers, the frequency of p53 mutation was reported in the literature to be in a range from $25 \%$ and up to $69 \%$ [3]. García, M.P.S et al. reported that p53 was epigenetically modified in $42 \%$ of OSCC[7]. However, studies reported that alteration in p53 function is mostly due to epigenetic events rather than genetic alteration[3]. Literature has discussed p53 hypermethylation as a marker with genomic instability in OSCC[7]. 


\begin{tabular}{|c|c|c|c|c|}
\hline Gene & Location & Function & Picture & References \\
\hline ECAD & CH16 & $\begin{array}{l}\text { Adhesive junction in the } \\
\text { epithelium. }\end{array}$ & & {$[14]$} \\
\hline DAPK & $\mathrm{CH} 9$ & Apoptosis & & [9] \\
\hline MGMT & $\mathrm{CH} 10$ & DNA repair & & {$[11]$} \\
\hline p53 & $\mathrm{CH} 7$ & $\begin{array}{l}\text { 1- } \text { Cell-cycle } \\
\text { progression. } \\
\text { 2- Cell } \\
\text { differentiation. } \\
\text { 3- DNA repair. } \\
\text { 4- Apoptosis. }\end{array}$ & & {$[3,12]$} \\
\hline
\end{tabular}

The detection of a gene methylation is considered a challenging process. DNA methylation analysis can be accomplished by several methods in the routine clinical practice[17]. Previous literatures has proven that the associations between DNA methylation patterns and clinical phenotypes can be used as biomarkers in diseases diagnosis and treatment[17]. For example, DNA methylation biomarkers have been shown to support clinical decisions in various cancers[17]. Methylated genes can serve as a promising, useful biomarker for new noninvasive OSCC detection and early diagnosis[2].

\section{Biomarker development and clinical applications}

To assess the methylation profile in cancerrelated genes several methods are available[18]. However, choosing the suitable method remains challenging[18]. DNA methylation biomarkers assay has many advantages that qualify them for extensive use and as screening tools in healthy individuals[17]. The fact that early diagnosis of OSCC can significantly improve clinical outcome[17].

DNA methylation analysis from saliva samples for epidemiological studies was reported in the literature where saliva sampling has several practical advantages over blood, since it is a non-invasive and easily reached to examine genetic modifications than venipuncture[19]. Also, saliva is in contact with the whole oral cavity and it is more convenient to be representative of the whole oral field when compared to localized tissue biopsy[19]. Recently, it is becoming more common to use DNA biomarkers in saliva to assess DNA methylation. However, $70 \%$ of DNA extracted from saliva is a mixture of bacterial DNA and the rest is from human DNA derived from epithelial and immune cells in the mouth[19]. Therefore, there are challenges to use salivary DNA in methylation studies that can influence data quality[19].

Bisulfite conversion (BSC) works on genomic DNA which is used in different techniques that help assessing the DNA methylation. These techniques require specific quantitative measures before and after BSC. Moreover, as a requirement for BSC, PCR amplification is needed which is known to be complicated. This is reported as PCR assay demands a specific methylated and non-methylated template generated by BCS[18].

DNA methylation and generally the epigenetic changes can play an important role in the early detection of individual's possibility to develop certain disease as well as early intervention by gene modification before disease developing and progression. A recent method is being discussed in the literatures is CRISPR technique. 


\section{CRISPR technique}

CRISPR technique is a newly reported method which has been discussed in the literature in the last three years[20]. This technique makes the gene sequence modification easier for the scientists[20]. It helps in quick changes of DNA on all organisms including human[20, 21]. As this technique is developed, revolution in biomedical researches is noted[21]. The advantages of this technique are: simple, time and cost efficient as well as results can be transmitted anywhere easily[21]. The aim of this technique is to correct human genes and reduce the risk of diseases[20, 21]. Using this technique may help in cancer treatment in an unusual manner. Also, it can reduce the risk of having genetic diseases. However, there is still some doubt regarding the safety and ethical issues for the human uses[21]. So, using CRISPR technique may help in identifying the genes that contribute to OSCC and correct them.

\section{CONCLUSION}

Due to the importance of gene contribution in OSCC, we reviewed the literature to discuss the most recently reported genes and to explore the new technologies which aid in the early diagnosis and treatment of OSCC aiming for a better prognosis. New technologies have been developed for this purpose, CRISPRS technique is a recent way that may aid to achieve such purpose.

\section{REFERENCES}

1. Imai, T., Toyota, M., Suzuki, H., Akino, K., Ogi, K., Sogabe, Y. (2008). Epigenetic inactivation of RASSF2 in oral squamous cell carcinoma. Cancer science, 99(5):958-66.

2. Dvojakovska, S., Popovic-Monevska, D., Grcev, A., Pancevski, G., Benedetti, A., Popovski, V.(2018). Promotor hypermethylated genes: Prospective diagnostic biomarkers in oral cancerogenesis. Journal of Cranio-Maxillofacial Surgery, 46(10):1737-40.

3. Hema, K., Smitha, T., Sheethal, H., Mirnalini, S.A.(2017). Epigenetics in oral squamous cell carcinoma. Journal of oral and maxillofacial pathology: JOMFP, 21(2):252.

4. Irimie, A.I., Ciocan, C., Gulei, D., Mehterov, N., Atanasov, A.G., Dudea, D.(2018). Current insights into oral cancer epigenetics. International journal of molecular sciences, 19(3):670.
5. Radhakrishnan, R., Kabekkodu, S., Satyamoorthy, K.(2011). DNA hypermethylation as an epigenetic mark for oral cancer diagnosis. Journal of Oral Pathology \& Medicine, 40(9):665-76.

6. Lindsay, C., Seikaly, H., Biron, V.L.(2017). Epigenetics of oropharyngeal squamous cell carcinoma: opportunities for novel chemotherapeutic targets. Journal of Otolaryngology-Head \& Neck Surgery, 46(1):9.

7. García, MP-S., García-García, A. (2012). Epigenome and DNA methylation in oral squamous cell carcinoma. Cancer Epigenetics: Springer, 207-19.

8. Folkman, J., Andrus, J.(2000). Tumor Angiogenesis in Cancer. Medicine,132-52.

9. Omim. (2015). Death-associated protein kinase 1; dapk1. 2015, March 25.

10. Hanahan, D., Weinberg, R.A. (2000). The hallmarks of cancer. Cell. 100(1):57-70.

11. GeneCards. (2019). MGMT Gene (Protein Coding).

12. OMIM.(2019). TUMOR PROTEIN p53; TP53. 2019, November 26.

13. Sporn, M.B.(1996). The war on cancer. Lancet (London, England), 347(9012):1377-81.

14. OMIM. Cadherin 1; cdh1.(2019). November 7 [Available from: https://www.omim.org/entry/192090?search=ECA D\&highlight=ecad.

15. Zakhari, S. (2013). Alcohol metabolism and epigenetics changes. Alcohol research: current reviews, 35(1):6.

16. Bouck, N., Stellmach, V., Hsu, S.C.(1996). How tumors become angiogenic. Advances in cancer research. 69: Elsevier, 135-74.

17. Bock, C., Halbritter, F., Carmona, F.J., Tierling, S., Datlinger, P., Assenov, Y.(2016). Quantitative comparison of DNA methylation assays for biomarker development and clinical applications. Nature biotechnology, 34(7):726.

18. Kurdyukov, S., Bullock, M. (2016). DNA methylation analysis: choosing the right method. Biology, 5(1):3.

19. Nishitani, S., Parets, S.E., Haas, B.W., Smith, A.K.(2018). DNA methylation analysis from saliva samples for epidemiological studies. Epigenetics, 13(4):352-62.

20. Ledford, H.(2015). Biohackers gear up for genome editing. Nature News, 524(7566):398.

21. Ledford, H.(2015). CRISPR, The disruptor. Nature News, 522(7554):20. 